University of Wollongong

Research Online

Faculty of Engineering and Information

Faculty of Engineering and Information

Sciences - Papers: Part A

Sciences

$1-1-2014$

New approach to improve the performance of fringe pattern profilometry using multiple triangular patterns for the measurement of objects in motion

Lei Lu

University of Wollongong, II490@uowmail.edu.au

Jiangtao Xi

University of Wollongong, jiangtao@uow.edu.au

Yanguang Yu

University of Wollongong, yanguang@uow.edu.au

Qinghua Guo

University of Wollongong, qguo@uow.edu.au

Follow this and additional works at: https://ro.uow.edu.au/eispapers

Part of the Engineering Commons, and the Science and Technology Studies Commons

Research Online is the open access institutional repository for the University of Wollongong. For further information contact the UOW Library: research-pubs@uow.edu.au 


\title{
New approach to improve the performance of fringe pattern profilometry using multiple triangular patterns for the measurement of objects in motion
}

\begin{abstract}
Fringe pattern profilometry using triangular patterns and intensity ratios is a robust and computationally efficient method in three-dimensional shape measurement technique. However, similar to other multipleshot techniques, the object must be kept static during the process of measurement, which is a challenging requirement for the case of fast-moving objects. Errors will be introduced if the traditional multiple-shot techniques are used directly in the measurement of a moving object. A new method is proposed to address this issue. First, the movement of the object is measured in real time and described by the rotation matrix and translation vector. Then, the expressions are derived for the fringe patterns under the influence of the two-dimensional movement of the object, based on which the normalized fringe patterns from the object without movement are estimated. Finally, the object is reconstructed using the existing intensity ratio algorithm incorporating the fringe patterns estimated, leading to improved measurement accuracy. The performance of the proposed method is verified by experiments.
\end{abstract}

\section{Keywords}

three-dimensional profile measurement, fringe patterns, intensity ratio

\section{Disciplines}

Engineering | Science and Technology Studies

\section{Publication Details}

L. Lu, J. Xi, Y. Yu \& Q. Guo, "New approach to improve the performance of fringe pattern profilometry using multiple triangular patterns for the measurement of objects in motion," Optical Engineering, vol. $53,(11)$ pp. 112211-1 - 112211-10, 2014. 


\section{Optical Engineering}

New approach to improve the performance of fringe pattern profilometry using multiple triangular patterns for the measurement of objects in motion

Lei Lu

Jiangtao Xi

Yanguang $\mathrm{Yu}$

Qinghua Guo 


\title{
New approach to improve the performance of fringe pattern profilometry using multiple triangular patterns for the measurement of objects in motion
}

\author{
Lei Lu, Jiangtao Xi, ${ }^{*}$ Yanguang $\mathrm{Yu}$, and Qinghua Guo \\ University of Wollongong, School of Electrical Computer and Telecommunications Engineering, Wollongong, New South Wales 2522, Australia
}

\begin{abstract}
Fringe pattern profilometry using triangular patterns and intensity ratios is a robust and computationally efficient method in three-dimensional shape measurement technique. However, similar to other multiple-shot techniques, the object must be kept static during the process of measurement, which is a challenging requirement for the case of fast-moving objects. Errors will be introduced if the traditional multiple-shot techniques are used directly in the measurement of a moving object. A new method is proposed to address this issue. First, the movement of the object is measured in real time and described by the rotation matrix and translation vector. Then, the expressions are derived for the fringe patterns under the influence of the two-dimensional movement of the object, based on which the normalized fringe patterns from the object without movement are estimated. Finally, the object is reconstructed using the existing intensity ratio algorithm incorporating the fringe patterns estimated, leading to improved measurement accuracy. The performance of the proposed method is verified by experiments. @ 2014 Society of Photo-Optical Instrumentation Engineers (SPIE) [DOI: 10.1117/1.OE.53.11.112211]
\end{abstract}

Keywords: three-dimensional profile measurement; fringe patterns; intensity ratio.

Paper 140168SS received Jan. 28, 2014; revised manuscript received Apr. 24, 2014; accepted for publication Apr. 29, 2014; published online May 22, 2014.

\section{Introduction}

As a noncontact three-dimensional (3-D) shape measurement technique, fringe pattern profilometry (FPP) has attracted intensive research over the past few decades. ${ }^{1-4}$ In contrast to other technologies, such as stereo vision, laser scanning, etc., FPP is advantageous by whole-field and reasonably high resolution. ${ }^{1,3}$ According to the number of fringe patterns used, FPP can be one-shot or multiple-shot. ${ }^{5}$ The one-shot techniques employ only a single image pattern to probe the object and, hence, are able to achieve fast measurement, but the accuracy suffers from the influence of ambient light and reflectivity variations. In order to achieve robust and accurate measurement, various multiple-shot techniques have been developed, e.g., phase shift profilometry (PSP) ${ }^{6-8}$ and intensity ratio approaches. ${ }^{9-13}$ In contrast to the one-shot techniques, these multiple-shot ones are more robust and able to achieve accurate measurement, but additional time is required due to the use of multiple image patterns. Besides, the object must be kept static during the projection and acquisition of the multiple image patterns. When the object moves during the measurement, errors will be introduced in the result of the measurement. The errors can be remedied by means of increasing the speed of digital projection and capture, ${ }^{14,15}$ which, however, usually leads to significant increases in the hardware cost.

For the multiple-shot techniques that are based on phase map, a number of approaches have been proposed to reduce the errors caused by the object movement. ${ }^{16-18}$ In particular, a method was presented in Ref. 18 to reduce the error following a number of steps. First, the information for the movement of the object is acquired in real time. Then analytical expressions are derived to describe the influence of movement on the fringe patterns. Finally, the analytical expressions are applied to the popular PSP algorithm, leading to a modified expression for the 3-D shape capable of achieving accurate measurement even when the object moves.

The intensity ratio approach was proposed by Jia et al. ${ }^{12,13}$ based on projection of multiple triangular patterns. Instead of calculating the phase map in the traditional phase shift profilometry, this technique reconstructs the object by computing the intensity ratio from the captured triangular fringe patterns. As the calculation of the intensity ratio does not involve the arctangent function, the computational burden of the intensity ratio is much less than the calculation of the phase map. ${ }^{11}$ However, similar to all other multipleshot techniques, the intensity ratio approach also requires that the object be kept static during the projection and acquisition of the multiple triangular patterns. When the object moves, errors will occur and there is no technique to tackle the problem yet.

Inspired by Ref. 18, in this paper, we propose a new approach to reduce the measurement error of intensity ratio approach due to the movement of the object. Similar to Ref. 18, we still consider the object is subject to a two-dimensional (2-D) movement, which can be described by a rotation matrix and translation vector. With the proposed method, the rotation matrix and translation vector are acquired in real time, which are incorporated into new expressions for the fringe patterns acquired from the object. These new expressions are then employed to form new formulation of the 3-D shape of the object in motion. 


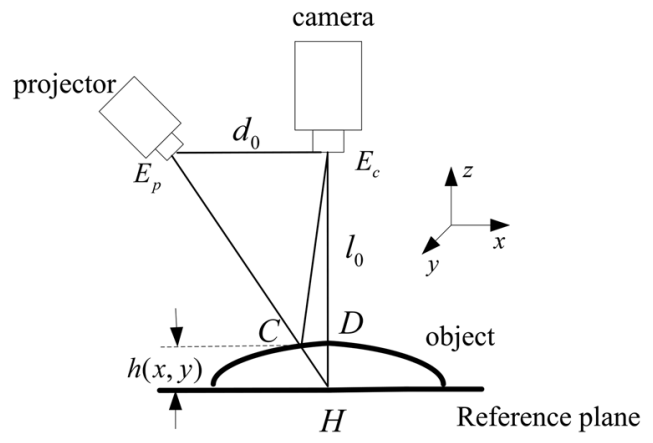

Fig. 1 The schematic diagram of the measurement system.

Experimental results are also presented to test the proposed approach.

This paper is organized as follows. Section 2 presents the principle of the intensity ratio approach. Section 3 is dedicated to describe the proposed technique. The effectiveness of the proposed method is verified by the experimental results in Sec. 4. Section 5 concludes the paper.

\section{Principle of the Intensity Ratio Approach}

A schematic diagram of the triangular pattern phase shifting profilometry is described in Fig. 1. For the sake of simplicity in terms of expressions, we assume that the camera is perpendicular to the reference plane.

With the intensity ratio approach, a set of (e.g., $N$ ) triangular fringe patterns is projected onto the reference plane and the object surface by the projector and captured by the camera. The fringe patterns have the same spatial period $T$, but are successively shifted by $T / N$. With these $N$ fringe patterns, the following is employed to yield a function called intensity ratio: ${ }^{19,20}$

$$
\begin{aligned}
r_{0}(x, y)= & \frac{\sum_{n=1}^{N}(-1)^{n+1} s_{n}(x, y)-\bmod (N, 2) \times s_{\min }(x, y)}{s_{\max }(x, y)-s_{\min }(x, y)} \\
& \times N \geq 2
\end{aligned}
$$

where $s_{n}(x, y)$ is the $n$ 'th highest intensity value among the $N$ projected patterns at the location of $(x, y) \cdot s_{\max }(x, y)$ and $s_{\min }(x, y)$ are the maximum and minimum intensities of the triangular patterns. Note that these fringe patterns are vertical strips, with the light intensity being constant in $y$ direction but varying in a triangular way in $x$ direction. It can be shown that $r_{0}(x, y)$ is still a triangular function with the period $T / N$, but its value is normalized into the range $[0,1]$ by Eq. (1). Then the following is applied to $r_{0}(x, y)$ :

$r_{T}(x, y)= \begin{cases}(-1)^{k+1} r_{0}(x, y)+2 \times \operatorname{round}\left(\frac{k-1}{2}\right) & k=1,2, \ldots, 2 N, N \text { is an odd number } \\ (-1)^{k} r_{0}(x, y)+2 \times \operatorname{round}\left(\frac{k}{2}\right)-1 & k=1,2, \ldots, 2 N, N \text { is an even number }\end{cases}$

where $k$ is called the region number, by which each fringe period $T$ is divided into $2 N$ equally spaced segments. With the operation in Eq. (2), we can obtain a function $r_{T}(x, y)$, which monotonically increases over every fringe period $T$. Then, $r_{T}(x, y)$ can be unwrapped to yield $r(x, y)$, which monotonically increases over the whole image pattern space. Note the above operations are applied to the fringe patterns from the object surface and the reference plane, yielding two unwrapped intensity ratios $r_{d}(x, y)$ and $r_{s}(x, y)$, respectively. lows:

At last, the height of the object can be calculated as fol-

$h(x, y)=\frac{\Delta r(x, y)}{T d_{0} / p l_{0}+\left(1 / l_{0}\right) \Delta r(x, y)}$,

where $h(x, y)$ is the height distribution of the object; $\Delta r(x, y)=r_{d}(x, y)-r_{s}(x, y)$ is the unwrapped intensity ratio difference between the object and reference plane; $d_{0}$ is the distance between the projector and camera; $l_{0}$ is the distance between the camera and the reference plane; $T$ is the fringe pitch of the pattern generated by a computer; and $p$ is the fringe pitch on the reference plane. Note that the parameters $d_{0}, l_{0}$, and $p$ can be determined by system calibration using a calibration board. ${ }^{21,22}$

Let us use an example where five triangular patterns are employed to show the procedure described above. Figure 2 shows the cross-section of the five triangular patterns on the reference plane. Figure 2(a) shows the intensity of the five spatial shifted triangular fringe patterns. $r_{0}(x, y)$ is still triangular with the period $T / 5$ but normalized into the range $[0,1]$, and $r_{T}(x, y)$ has its value increasing from 0 to 10 over the period $T$.

The performance of the above depends on whether Eqs. (1) and (2) yield a function monotonically increasing over every fringe period $T$. This requires not only accurate creation and projection of the fringe patterns, but also keeping the object static during the measurement. Apparently, when the object moves, measurement error will occur.

\section{Proposed Approach}

In order to reduce the error caused by object movement, we need to figure out how the movement influences the fringe patterns acquired from the object surface. Assuming that the object is subject to a 2-D movement on the $x-y$ plane, a point $(x, y)$ on the object surface moves to the point $(u, v)$ following the relationship below:

$$
\left[\begin{array}{l}
x \\
y
\end{array}\right]=\mathbf{R}\left[\begin{array}{l}
u \\
v
\end{array}\right]+\mathbf{T}, \quad\left[\begin{array}{l}
u \\
v
\end{array}\right]=\overline{\mathbf{R}}\left[\begin{array}{l}
x \\
y
\end{array}\right]+\overline{\mathbf{T}},
$$

where $R, \bar{R}, T$, and $\bar{T}$ are referred to as rotation matrixes and translation vectors, which describe the relationship between $(x, y)$ and $(u, v)$, and they are given by

$$
\mathbf{R}=\left[\begin{array}{ll}
r_{11} & r_{12} \\
r_{21} & r_{22}
\end{array}\right], \quad \mathbf{T}=\left[\begin{array}{l}
t_{1} \\
t_{2}
\end{array}\right],
$$



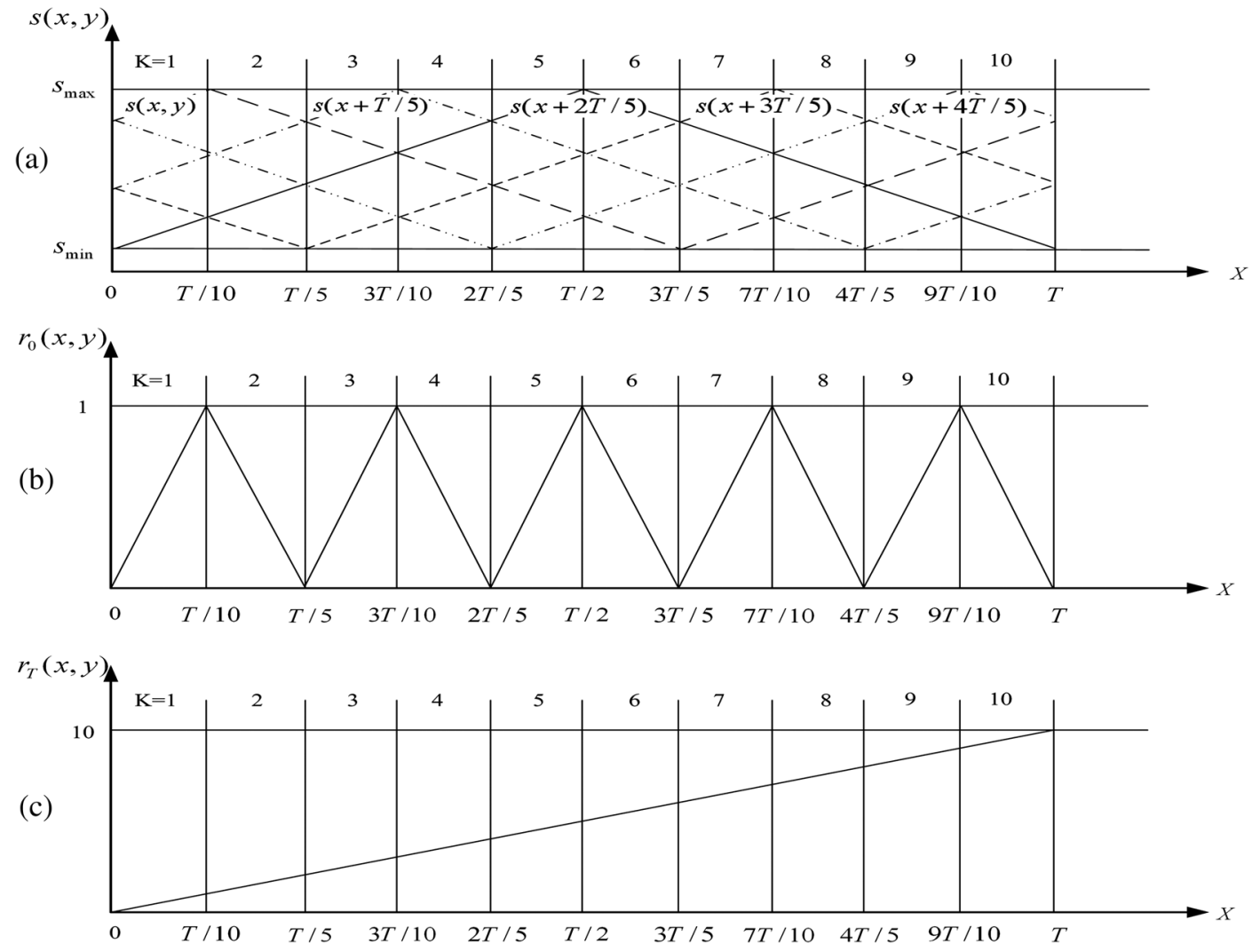

Fig. 2 Five-step triangular pattern phase shifting method: (a) cross-section of the five triangular patterns shifted by $T / 5$ of the fringe period; (b) cross-section of the triangular shape intensity ratio; (c) cross-section of the intensity ratio ramp after removal of triangles.

$\overline{\mathbf{R}}=\left[\begin{array}{ll}\bar{r}_{11} & \bar{r}_{12} \\ \bar{r}_{21} & \bar{r}_{22}\end{array}\right], \quad \overline{\mathbf{T}}=\left[\begin{array}{l}\bar{t}_{1} \\ \bar{t}_{2}\end{array}\right]$.

The relationship between $(\mathbf{R}, \mathbf{T})$ and $(\overline{\mathbf{R}}, \overline{\mathbf{T}})$ can be expressed as

$\overline{\mathbf{R}}=\mathbf{R}^{-1}, \quad \overline{\mathbf{T}}=-\mathbf{R}^{-1} \mathbf{T}$.

As the shape of the object surface does not change during the movement, according to Ref. 18, the height distribution of the object surface with movement becomes

$\tilde{h}_{x-y}(u, v)=h_{x-y}(x, y)=h_{x-y}[f(u, v), g(u, v)]$,

where the subscript $x-y$ denotes the coordinate system in which the functions are defined, and from Eq. (5), we have

$$
\begin{aligned}
& f(u, v)=r_{11} u+r_{12} v+t_{1}, \\
& g(x, y)=r_{21} u+r_{22} v+t_{2} .
\end{aligned}
$$

Without loss of generality, $(u, v)$ can be replaced by $(x, y)$, yielding the following:

$$
\tilde{h}_{x-y}(x, y)=h_{x-y}[f(x, y), g(x, y)] \text {. }
$$

Assume $s(x, y)$ is one triangular fringe pattern from the reference plane and the corresponding fringe pattern on the object can be described by $d(x, y)$. According to the model described in Ref. 23, the deformed fringe pattern $d(x, y)$ is a shifted version of $s(x, y)$. $d(x, y)=s[x-u(x, y), y]$,

where $u(x, y)$ is the shift function, which varies with the height of the object. The height distribution of the object is given by

$h(x, y)=\frac{l_{0} u(x, y)}{u(x, y)+d_{0}}$.

Corresponding to Eq. (11), the fringe patterns of the object with movement can be expressed as follows:

$\tilde{d}_{x-y}(x, y)=s[x-\tilde{u}(x, y), y]$,

where $\tilde{d}_{x-y}(x, y)$ is the fringe pattern of object with movement and $\tilde{u}(x, y)$ is the shift function of object with movement. From Eq. (12) we can see a corresponding relationship between the height distribution and the shift function, and hence, a similar relationship to Eq. (10) should also hold for the shift function without and with movement.

$\tilde{u}(x, y)=u[f(x, y), g(x, y)]$

Substituting Eq. (14) into Eq. (13) yields the following:

$\tilde{d}_{x-y}(x, y)=s\{x-u[f(x, y), g(x, y)], y\}$.

Note Eq. (15) is defined in $x-y$ coordinate system. Now let us consider Eq. (15) in a new coordinate system $\xi-\eta$, which has the relationship to the $x-y$ system as follows: 
$\left[\begin{array}{l}x \\ y\end{array}\right]=\overline{\mathbf{R}}\left[\begin{array}{l}\xi \\ \eta\end{array}\right]+\overline{\mathbf{T}}$.

In $\xi-\eta$ coordinate system, Eq. (15) becomes

$$
\begin{aligned}
\tilde{d}_{\xi-\eta}(\xi, \eta) & =\tilde{d}_{x-y}[\bar{f}(\xi, \eta), \bar{g}(\xi, \eta)] \\
& =s[\bar{f}(\xi, \eta)-u(\xi, \eta), \bar{g}(\xi, \eta)],
\end{aligned}
$$

where from Eq. (6) we have

$\bar{f}(\xi, \eta)=\bar{r}_{11} \xi+\bar{r}_{12} \eta+\bar{t}_{1}, \quad \bar{g}(\xi, \eta)=\bar{r}_{21} \xi+\bar{r}_{22} \eta+\bar{t}_{2}$.

Equation (17) is the expression of the fringe patterns in the $\xi-\eta$ coordinate system. When $(\overline{\mathbf{R}}, \overline{\mathbf{T}})$ are available, $\tilde{d}_{\xi-\eta}(\xi, \eta)$ can be obtained. As Eq. (17) holds for arbitrary 2-D movement, it can be rewritten in a general form as follows:

$\tilde{d}(x, y)=s[\bar{f}(x, y)-u(x, y), \bar{g}(x, y)]$.

Equation (19) reveals the influence of the object movement on the fringe patterns. Obviously, direct use of these patterns in the intensity ratio method in Eqs. (1) and (2) will result in measurement error. However, when $\bar{f}(x, y)$ and $\bar{g}(x, y)$ are available, Eq. (19) can also be utilized to estimate the fringe patterns without movement. Instead of the fringe patterns acquired, these estimated fringe patterns will be used to compute the intensity ratios, which will lead to reduction of the measurement error. This is the basic idea of the proposed approach.

In order to estimate the fringe patterns without movement, we first normalize the fringe patterns from the reference plane based on the peak points $s_{\max }(x, y)$ and the bottom points $s_{\min }(x, y)$. Figure 3(a) shows an example of the normalized fringe pattern $s_{0}(x, y)$ with its value falling within the range from 0 to 1 . Then we convert the normalized pattern into a monotonically increasing one, $s_{r}(x, y)$, as shown in Fig. 3(b). $s_{r}(x, y)$ ranges from 0 to 2. Finally, we unwrap the results to yield the form monotonically increasing over the whole image space, as shown in Fig. 3(c).

Because the fringe patterns on the object and reference plane satisfy Eqs. (11) and (19), the unwrapped fringe patterns should also satisfy the same relationship. As $s_{l}(x, y)$ is a linear function with respect to $x$, we have

$d_{l}(x, y)=s_{l}[x-u(x, y), y]=s_{l}(x, y)+s_{l}[-u(x, y), y]$,

$\tilde{d}_{l}(x, y)=s_{l}[\bar{f}(x, y)-u(x, y), \bar{g}(x, y)]=s_{l}[\bar{f}(x, y)$,

$\bar{g}(x, y)]+s_{l}[-u(x, y), \bar{g}(x, y)]$,

where $d_{l}(x, y)$ and $\tilde{d}_{l}(x, y)$ are the unwrapped fringe patterns of object without movement and with movement, respectively.

For the vertical fringe patterns used, the intensity values are constant with respect to $y$ direction.

$s_{l}[-u(x, y), y]=s_{l}[-u(x, y), \bar{g}(x, y)]$.

Substituting Eqs. (20) and (22) into Eq. (21), we have the following:
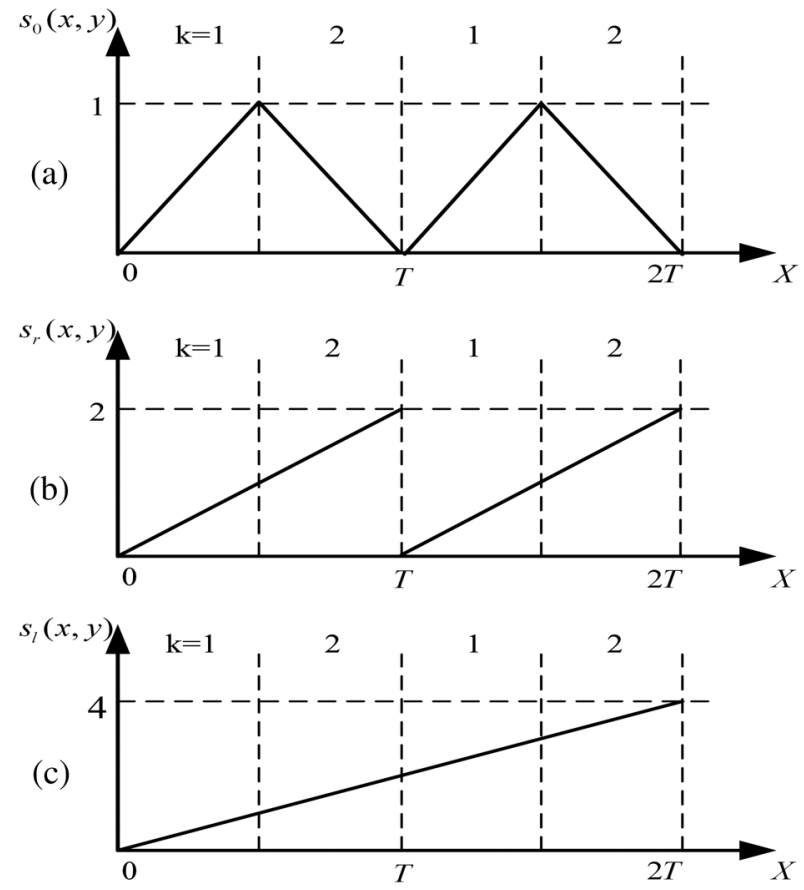

Fig. 3 Transform of the triangular fringe patterns: (a) cross-section of the normalized triangular fringe patterns; (b) cross-section of the fringe pattern ramp; (c) cross-section of the unwrapped fringe patterns.

$d_{l}(x, y)=\tilde{d}_{l}(x, y)-s_{l}[\bar{f}(x, y), \bar{g}(x, y)]+s_{l}(x, y)$.

Equation (23) can be used to estimate the unwrapped fringe patterns from the object without movement.

In order to have the triangular fringe patterns required for computation of intensity ratio, $d_{l}(x, y)$ is wrapped based on the inverse processes shown in Fig. 3, yielding estimation of the normalized triangular fringe patterns of object without movement $d_{0}(x, y)$.

In summary, based on the derivation above, the proposed approach can be implemented by the following steps:

Step 1: Project $N$ fringe patterns on to the surface of the object and then normalize and unwrap the fringe patterns acquired from both the object surface and the reference plane.

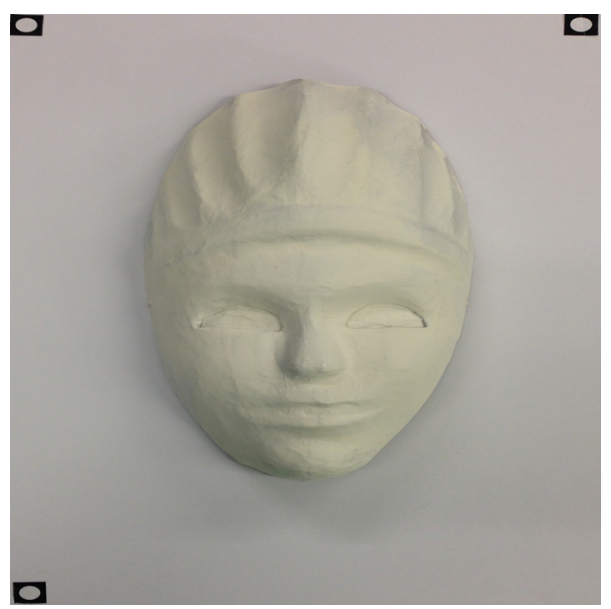

Fig. 4 The mask with the circle marks. 


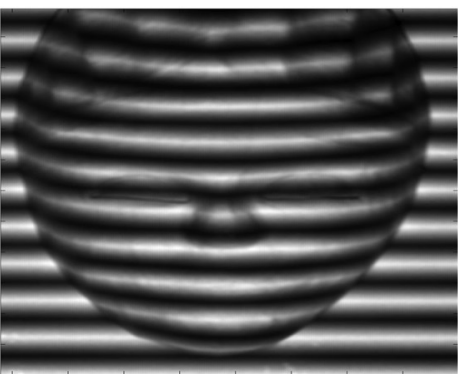

(a)

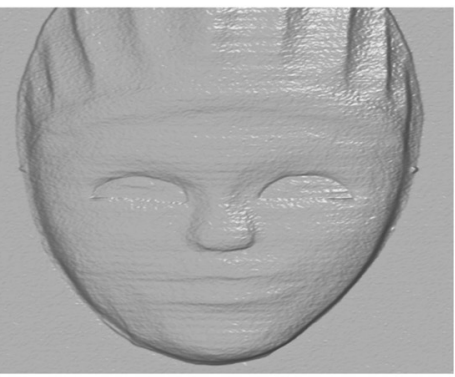

(b)

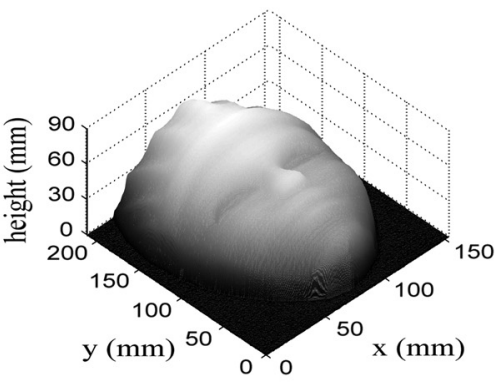

(c)

Fig. 5 Reconstructed result of the traditional triangular pattern phase shifting profilometry when the object is static: (a) the triangular fringe patterns of the first step; (b) the front view of the reconstructed result of the object; (c) the mesh display of the reconstructed result of the object.

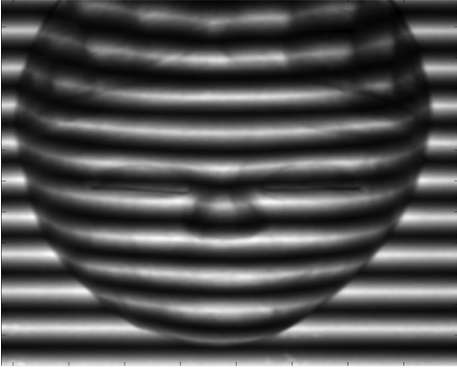

(a)

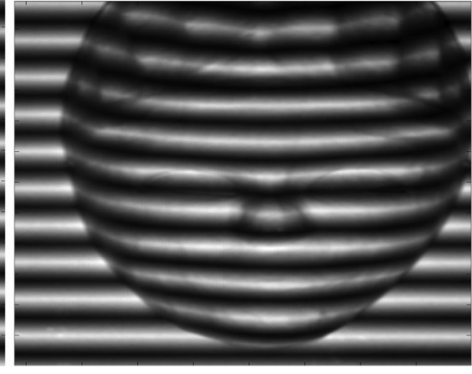

(b)

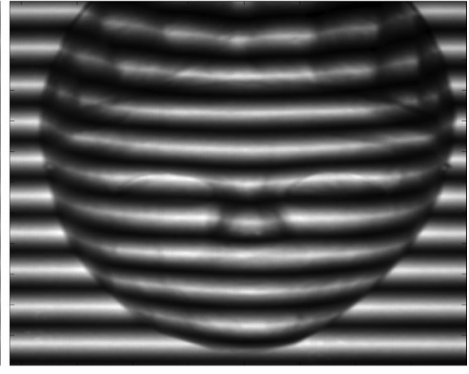

(c)

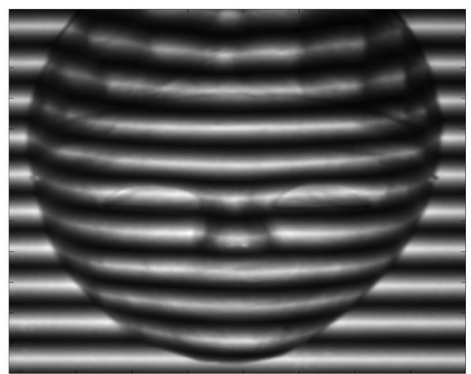

(d)

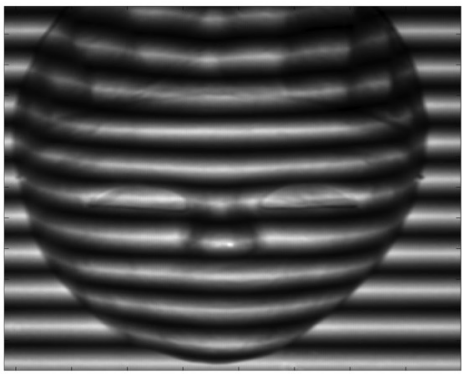

(e)

Fig. 6 The captured triangular fringe patterns when the object has oblique movement: (a) to (e) the fringe patterns of object from the first step to the fifth step.

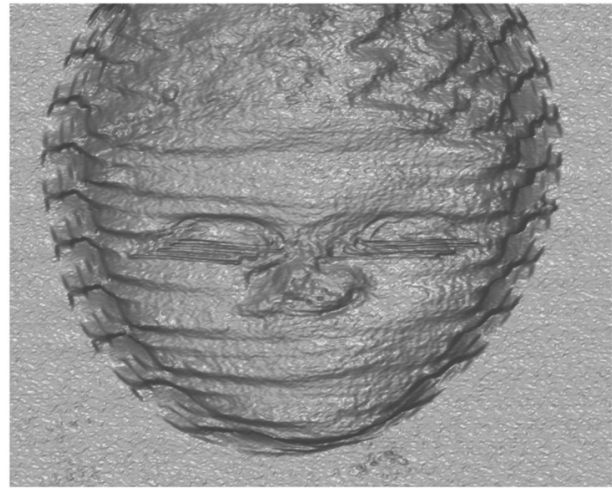

(a)

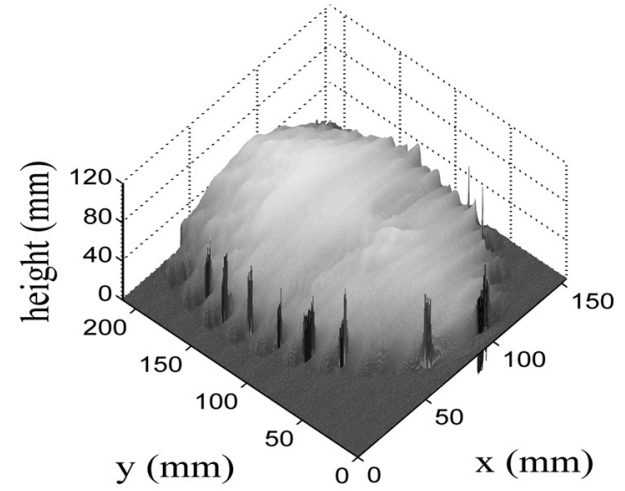

(b)

Fig. 7 The reconstructed result of the traditional triangular pattern phase shifting profilometry when the object has oblique movement: (a) the front view of the reconstructed result; (b) the mesh display of the reconstructed result. 


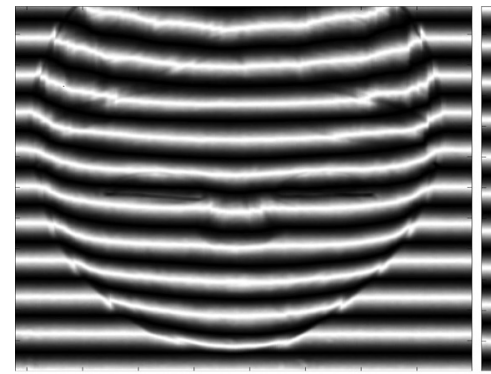

(a)

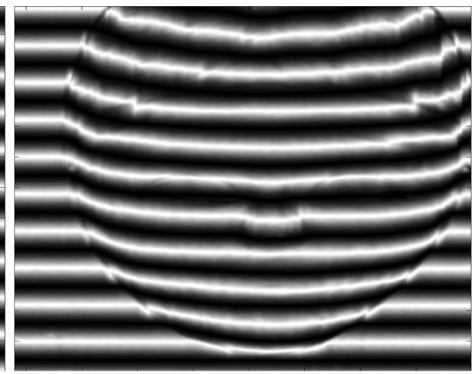

(b)

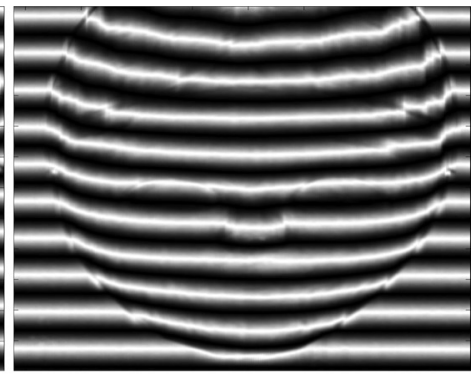

(c)

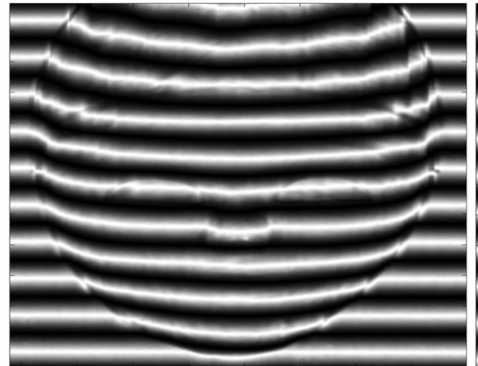

(d)

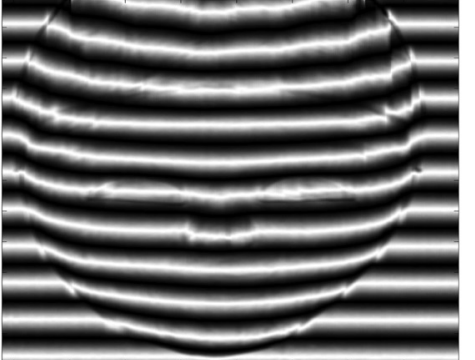

(e)

Fig. 8 The normalized fringe patterns of object with movement: (a) to (e) the normalized fringe patterns of the object with movement from the first step to the fifth step.

Step 2: Calculate the rotation matrixes and translation vectors for the movement of object when each fringe pattern captured. Note that we can use the same approach as described in Ref. 18.

Step 3: With the rotation matrix and translation vector, use Eq. (23) to determine the estimation of the unwrapped fringe patterns from the object without movement.

Step 4: Construct the estimation of triangular patterns from the object without movement.
Step 5: Construct the 3-D shape of the object using intensity ratio-to-shape conversion relationship in Eq. (3).

\section{Experiments}

The experiment system includes a projector (Hitachi CPX260) with the resolution of $1024 \times 768$ and a camera (Duncan Tech MS3100) with a resolution of $1392 \times 1039$. The system parameters $d_{0}$ and $l_{0}$ are calibrated as 328.9 and $1280.0 \mathrm{~mm}$; the fringe pitch on the reference plane $p$

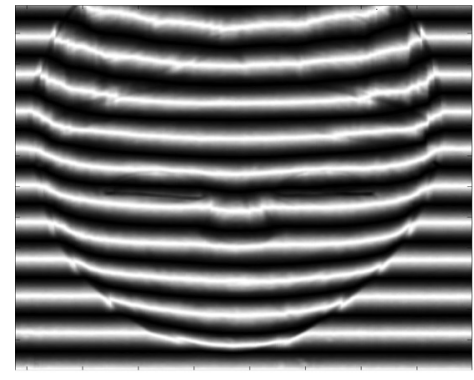

(a)

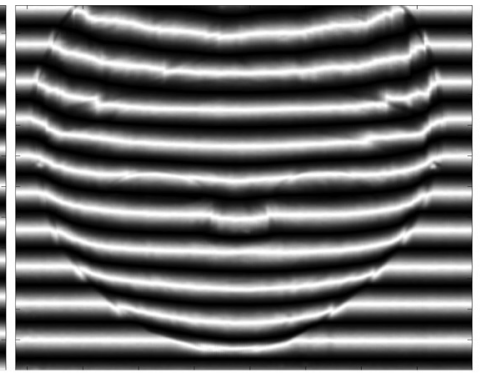

(b)

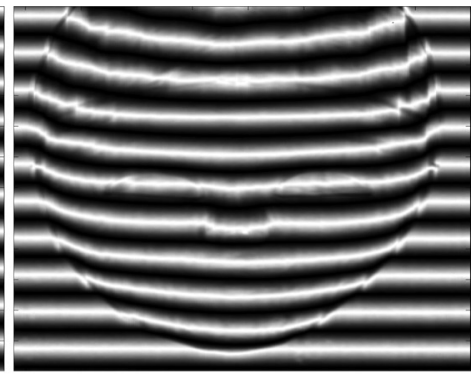

(c)

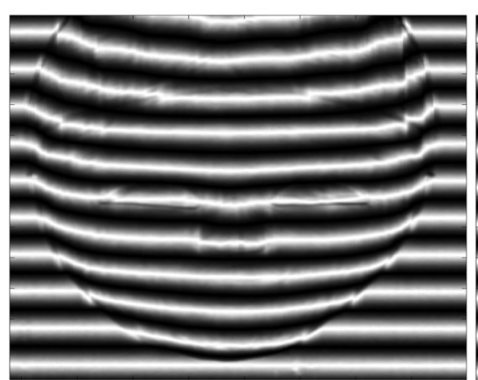

(d)

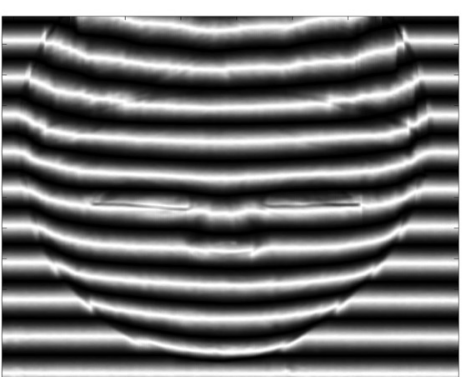

(e)

Fig. 9 The estimated normalized fringe patterns of the object without movement: (a) to (e) the normalized fringe patterns of the object without movement from the first step to the fifth step. 


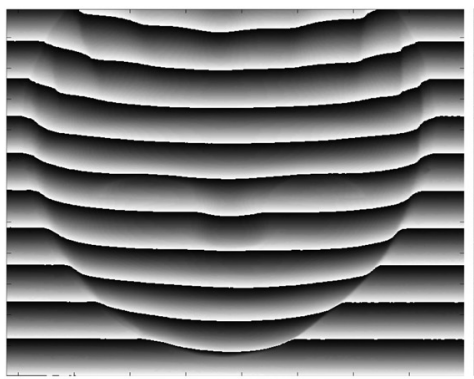

(a)

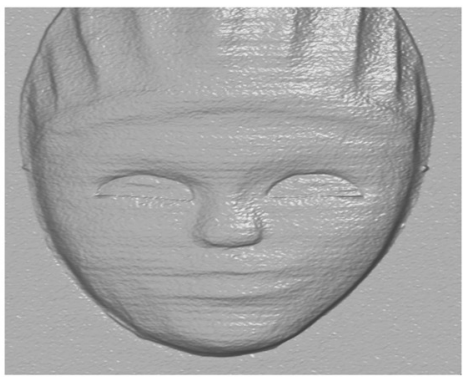

(b)

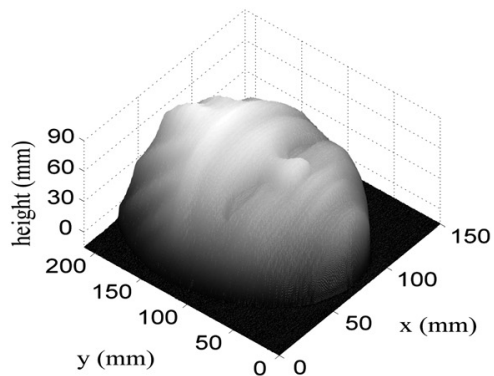

(c)

Fig. 10 The reconstructed results with the proposed algorithm when the object has oblique movement: (a) the wrapped intensity ratio of the object; (b) the front view of the reconstructed result; (c) the mesh display of the reconstructed mask.

is $22.597 \mathrm{~mm}$ per period. A mask is measured to verify the proposed algorithm in the experiment. The size of the mask is $\sim 130 \times 280 \mathrm{~mm}$, and a five-step intensity ratio method is used to reconstruct the object. Similar to the method described in Ref. 18, three circle marks as shown in Fig. 4 are used to calculate the rotation matrix and translation vector. When the multiple fringe patterns are captured, the locations of the center of the circle marks are extracted, which are used as the corresponding points in the leastsquares fitting algorithm described in Ref. 24 for determining the rotation matrix and translation vector.

In the first experiment, the traditional five-step intensity ratio method described in Ref. 13 is used and the object is kept static, and the reconstructed 3-D shape is shown in Fig. 5. Figure 5(a) is the triangular fringe pattern for the first step. Figure 5(b) is the front view of the reconstructed result, and Fig. 5(c) is the mesh display of the result. The results clearly show that the traditional intensity ratio method works well when the object is kept static.
In the second experiment, we still use the traditional intensity ratio method, but the object moves obliquely during the projection of the five successive fringe patterns. Figure 6 shows the five fringe patterns captured from the object. The object is moved to the right direction for $7.7 \mathrm{~mm}$ in the second step, $4.3 \mathrm{~mm}$ in the left direction in the third step, and 3.5 and $2.7 \mathrm{~mm}$ in the left and down direction in the fourth and fifth steps. The reconstructed results for the second experiment are shown in Fig. 7. Figure 7(a) is the front view of the reconstructed result, and Fig. 7(b) is the mesh display of the result, which is significantly distorted.

Then, in the third experiment, we applied the proposed method to the object moving in the same way as above. Figure 8 shows the normalized fringe patterns acquired from the object in motion.

With the proposed method, we are able to obtain estimations of the normalized fringe patterns from the object without movement, as shown in Fig. 9. It is seen that, compared with the positions of the object in Fig. 8, the object is moved back by the proposed method.

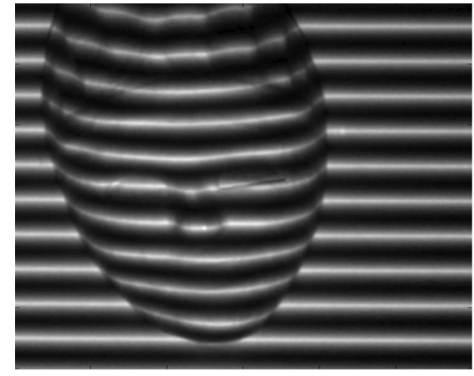

(a)

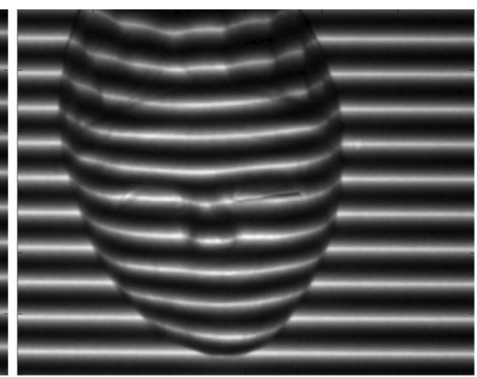

(b)

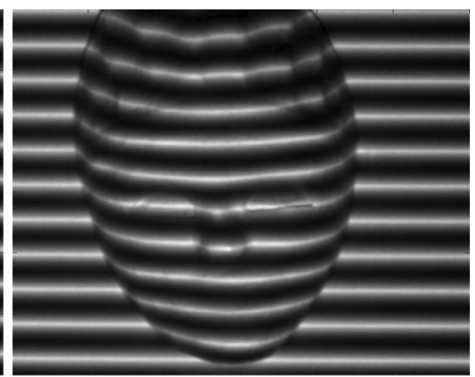

(c)

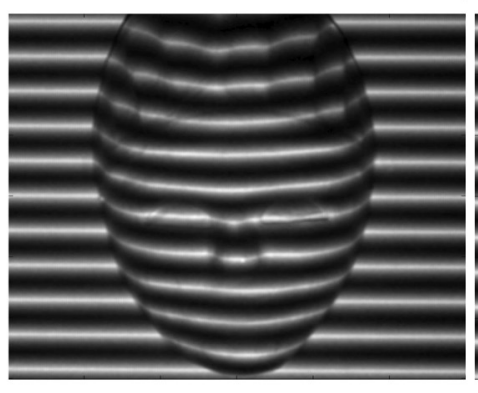

(d)

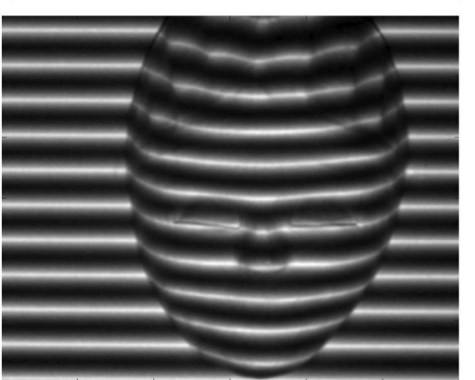

(e)

Fig. 11 The captured triangular fringe patterns when the object has rotation movement: (a) to (e) the fringe patterns of the object from the first step to the fifth step. 


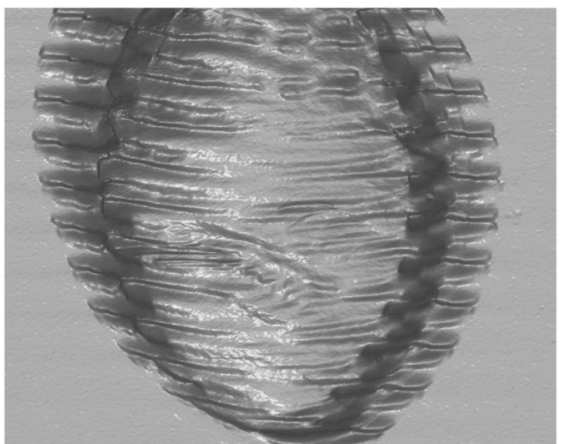

(a)

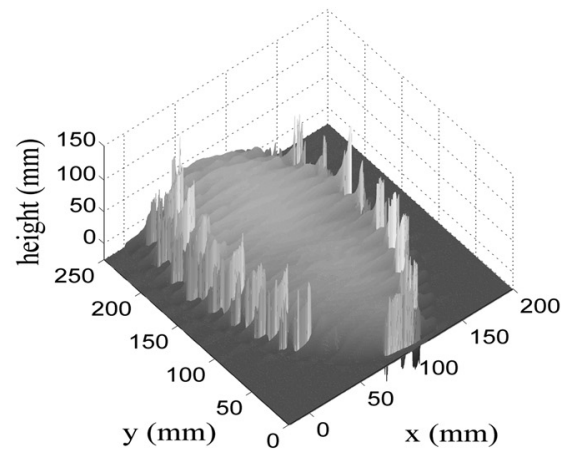

(b)

Fig. 12 The reconstructed result of the traditional triangular pattern phase shifting profilometry when the object has rotation movement: (a) the front view of the reconstructed result; (b) the mesh display of the reconstructed result.

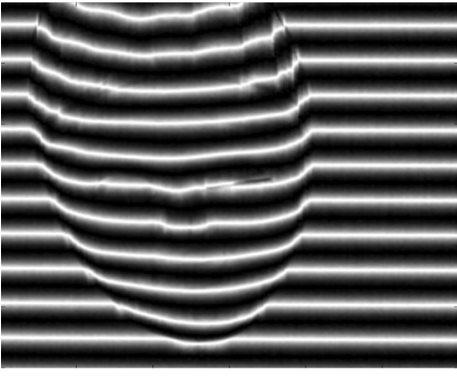

(a)

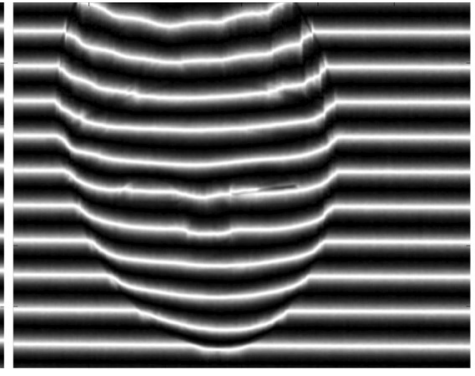

(b)

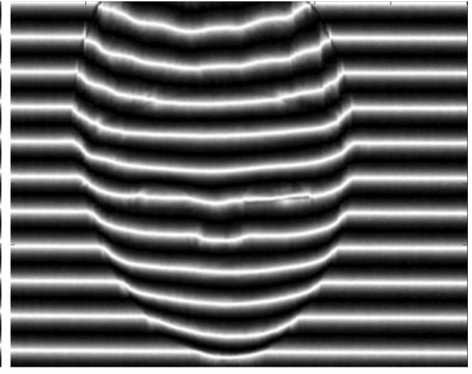

(c)

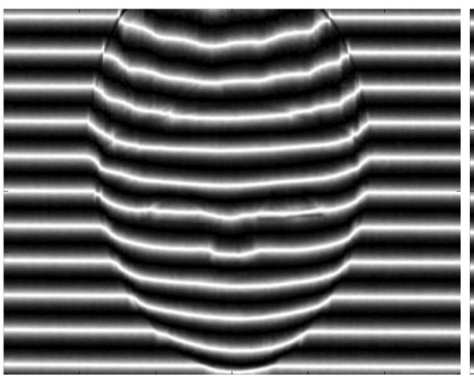

(d)

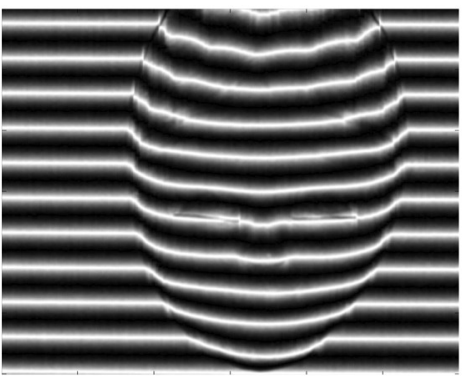

(e)

Fig. 13 The normalized fringe patterns of object with the rotation movement: (a) to (e) the normalized fringe patterns of the object with movement from the first step to the fifth step.

Until now, the normalized fringe patterns of the object without movement are obtained, which then can be used in the traditional intensity ratio method with the results shown in Fig. 10. Figure 10(a) is the wrapped intensity ratio of the mask; Fig. 10(b) is the front view of the reconstructed result; and Fig. 10(c) is the mesh display of the result. Within contrast to the results in Fig. 7, the surface of the mask is well reconstructed and, hence, significant improvement is achieved.

In the fourth experiment, we consider the case when the object is rotated clockwise around the bottom left corner during the measurement. The rotation angle is $0.1671,0.1446$, 0.1356 , and $0.1103 \mathrm{rad}$ for the second step, third step, fourth step, and fifth step, respectively. Figure 11 shows the captured fringe patterns of object from the first step to the fifth step.

Figure 12 shows the reconstructed results obtained by the traditional intensity ratio method, which are obviously distorted in contrast to the original mask.
Then, the proposed approach is applied when the object is subject to the same rotation in the fifth experiment. Figure 13 shows the normalized fringe patterns of object with movement from the first step to the fifth step. With the proposed approach, the fringe patterns of the object without movement can be estimated, as shown in Fig. 14. With the estimation of the normalized fringe patterns from the object without movement, the 3-D shape of the object is reconstructed by the traditional intensity ratio technique. As shown in Fig. 15, the results are much better than those in Fig. 12.

Table 1 shows the root mean square (RMS) error for the experimental results presented above, with the measurement result in Fig. 5 (the mask is kept static) as the reference. It is seen that, without the proposed method, the RMS error is 63.559 and $89.251 \mathrm{~mm}$, respectively. When the proposed approach is employed, the RMS error becomes 0.381 and $0.426 \mathrm{~mm}$. Hence, we can say that accuracy can be improved significantly by the proposed method. 


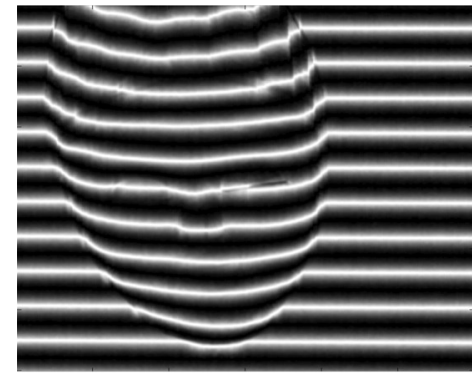

(a)

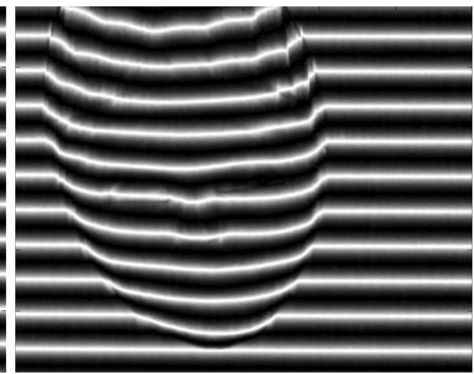

(b)

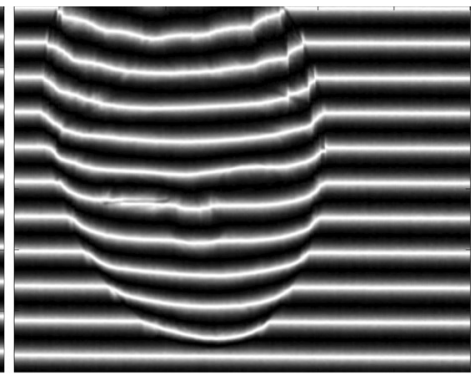

(c)

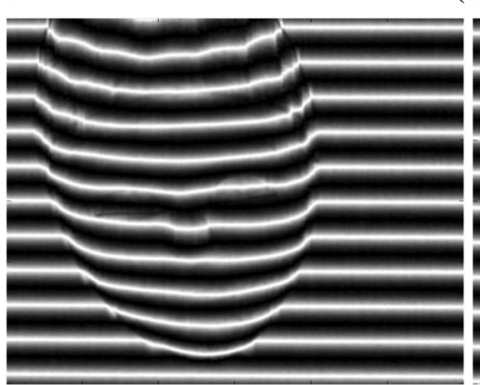

(d)

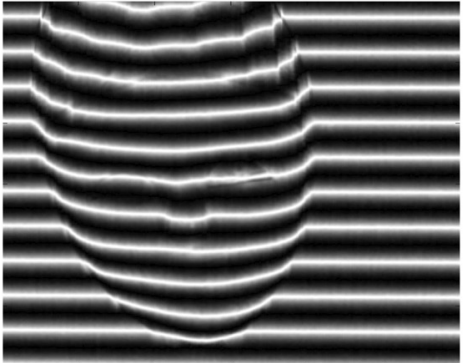

(e)

Fig. 14 The estimated normalized fringe patterns of the object without movement: (a) to (e) the normalized fringe patterns of the object without movement from the first step to the fifth step.

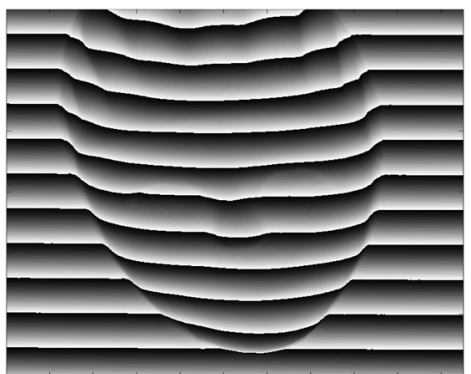

(a)

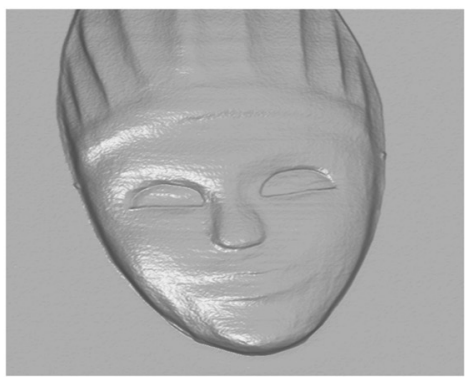

(b)

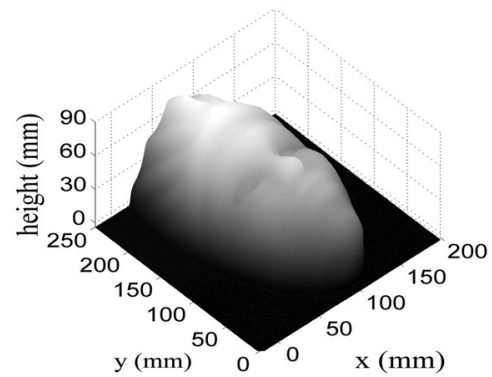

(c)

Fig. 15 The reconstructed results with the proposed algorithm when the object has rotation movement: (a) the wrapped intensity ratio of the object; (b) the front view of the reconstructed result; (c) the mesh display of the reconstructed mask.

Table 1 The root mean square (RMS) measurement error of the mask.

\begin{tabular}{lcc}
$\begin{array}{l}\text { Movement } \\
\text { type }\end{array}$ & $\begin{array}{c}\text { RMS error (the traditional } \\
\text { triangular pattern } \\
\text { profilometry) }\end{array}$ & $\begin{array}{c}\text { RMS error (the } \\
\text { proposed } \\
\text { algorithm) }\end{array}$ \\
\hline Oblique & $63.559 \mathrm{~mm}$ & $0.381 \mathrm{~mm}$ \\
Rotation & $89.251 \mathrm{~mm}$ & $0.426 \mathrm{~mm}$ \\
\hline
\end{tabular}

\section{Conclusion}

In this paper, an approach has been proposed to improve the accuracy of 3-D measurement of moving objects based on triangular patterns and intensity ratio method. The proposed method consists of the following steps. First, the fringe patterns of the moving object are captured. Then the rotation matrix and translation vector are calculated to describe the movement of the object. Based on analysis of the influence of the movement on the fringe patterns, normalized fringe patterns of object without movement can be estimated, which are utilized to compute the intensity ratio for the construction of the 3-D shape of the object. The performance of the proposed method has been confirmed by a series of experiments.

\section{References}

1. S. Zhang, "Recent progresses on real-time 3-D shape measurement using digital fringe projection techniques," Opt. Lasers Eng. 48(2), 149-158 (2010)

2. Y. Ding et al., "Recovering the absolute phase maps of two fringe patterns with selected frequencies," Opt. Lett. 36(13), 2518-2520 (2011).

3. S. S. Gorthi and P. Rastogi, "Fringe projection techniques: whither we are?," Opt. Lasers Eng. 48(2), 133-140 (2010).

4. Y. Hu et al., "Blind color isolation for color-channel-based fringe pattern profilometry using digital projection," J. Opt. Soc. Am. A 24(8), 2372-2382 (2007).

5. Z. Zhang, "Review of single-shot 3D shape measurement by phase calculation-based fringe projection techniques," Opt. Lasers Eng. 50(8), 1097-1106 (2012). 
6. H. Liu, V. Srinivasan, and M. Halioua, "Automated phase-measuring profilometry of 3-D diffuse objects," Appl. Opt. 23(18), 3105-3108 (1984).

7. X. Su and W. Zhou, "Automated phase-measuring profilometry using defocused projection of a Ronchi grating," Opt. Commun. 94(6), 561573 (1992).

8. H. Su, J. Li, and X. Su, "Phase algorithm without the influence of carrier frequency," Opt. Eng. 36(6), 1799-1805 (1997).

9. B. Carrihill and R. Hummel, "Experiments with the intensity ratio depth sensor," Comput. Vis. Graph. Image Process. 32(3), 337-358 (1985).

10. T. Miyasaka and K. Araki, "Development of real time 3-D measurement system using intensity ratio method," in Proc. ISPRS Commission III, Photogrammetric Computer Vision (PCV02), Vol. 34(3B), pp. 181-185 (2002).

11. P. Huang, S. Zhang, and F. Chiang, "Trapezoidal phase-shifting method for three-dimensional shape measurement," Opt. Eng. 44(12), 123601 (2005)

12. P. Jia et al., "Two-step triangular-pattern phase-shifting method for three-dimensional object shape measurement," Opt. Eng. 46(8), 083201 (2007)

13. P. Jia, J. Kofman, and C. English, "Multiple-step triangular-pattern phase shifting and the influence of number of steps and pitch on measurement accuracy," Appl. Opt. 46(16), 3253-3262 (2007).

14. P. Huang and S. Zhang, "Fast three-step phase-shifting algorithm," Appl. Opt. 45(21), 5086-5091 (2006).

15. B. Li et al., "Some recent advances on superfast 3D shape measurement with digital binary defocusing techniques," Opt. Lasers Eng. 54(7), 236-246 (2014).

16. S. Zhang and S. T. Yau, "High-speed three-dimensional shape measurement system using a modified two-plus-one phase-shifting algorithm," Opt. Eng. 46(11), 113603 (2007).

17. E. Hu and Y. He, "Surface profile measurement of moving objects by using an improved $\pi$ phase-shifting Fourier transform profilometry," Opt. Lasers Eng. 47(1), 57-61 (2009).

18. L. Lu et al., "New approach to improve the accuracy of 3-D shape measurement of moving object using phase shifting profilometry," Opt. Express 21(25), 30610-30622 (2013).

19. K. Wu et al., "3D profile measurement based on estimation of spatial shifts between intensity ratios from multiple-step triangular patterns," Opt. Lasers Eng. 51(4), 440-445 (2013).

20. Z. Yang et al., "Intensity ratio approach for 3D profile measurement based on projection of triangular patterns," Appl. Opt. 53(2), 200-207 (2014).

21. Z. Zhang, "A flexible new technique for camera calibration," IEEE Trans. Pattern Anal. Mach. Intell. 22(11), 1330-1334 (2000).
22. S. Zhang and P. Huang, "Novel method for structured light system calibration," Opt. Eng. 45(8), 083601 (2006).

23. Y. Hu et al., "Study on generalized analysis model for fringe pattern profilometry," IEEE Trans. Instrum. Meas. 57(1), 160-167 (2008).

24. K. S. Arun, T. S. Huang, and S. D. Blostein, "Least-squares fitting of two 3-D point sets," IEEE Trans. Pattern Anal. Mach. Intell. PAMI-9 (5), 698-700 (1987).

Lei Lu received BS from Henan University of Science and Technology, China in 2007, and MS degree from the Zhengzhou University, China in 2011. Now he is the PhD candidate in University of Wollongong.

Jiangtao $\mathbf{X i}$ is a professor at the University of Wollongong. He received his BS from Beijing Institute of Technology, China, in 1982, his MS degree from TsingHua University, China, in 1985, and his PhD degree from University of Wollongong, Australia, in 1996. He was a lecturer and associate professor in Zhengzhou University, China, from 1985 to 1991; a postdoctoral fellow at McMaster University, Canada, in 1995; at Bell Laboratories from 1996 to 1998; and the chief technical officer of TCL IT Group Co. Ltd., China, from 2000 to 2002.

Yanguang $\mathbf{Y u}$ is a senior lecturer in University of Wollongong. She received $\mathrm{BE}$ from Huazhong University of Science and Technology, China (1986) and PhD degree from Harbin Institute of Technology, China (2000). She was a postdoctoral fellow in Tianjin University, China (2001-2002); visiting fellow at University of Pavia, Italy (2002-2003); visiting fellow at University of Wollongong, Australia (2004-2005); visiting associate professor and professor at the Engineering School ENSEEIHT of Toulouse, France (2004 and 2006).

Qinghua Guo received BE in electronic engineering and ME in signal and information processing from Xidian University in 2001 and 2004, respectively, and $\mathrm{PhD}$ in electronic engineering from City University of Hong Kong in 2008. He is currently with the University of Wollongong. Dr Guo is a recipient of the Australian Research Council's inaugural DECRA. 